Canadian Oncology

Nursing Journal

Revue canadienne

de soins infirmiers

en oncologie

Volume 27, Issue 2 • Spring 2017

elSSN: 2368-8076 


\title{
Rôles joués par les infirmières pivots en oncologie pour accroître la responsabilisation des adultes atteints d'un cancer du poumon durant la phase diagnostique
}

\author{
par Gaya Jeyathevan, Manon Lemonde et Angela Cooper Brathwaite
}

\section{RÉSUMÉ}

But : Le but de cette étude était d'explorer les rôles joués par les infirmières pivots en oncologie (IPO) pour accroître la responsabilisation des adultes atteints d'un cancer du poumon durant la phase diagnostique des soins oncologiques.

Devis : Une étude descriptive qualitative a été utilisée. Les données proviennent d'entrevues individuelles avec des patients et d'un groupe de discussion formé d'IPO.

Résultats : Huit personnes ont participé : quatre (4) adultes atteints d'un cancer du poumon et quatre (4) infirmières pivots en oncologie. Les thèmes liés aux fonctions cliniques des rôles joués par l'IPO pour accroître la responsabilisation des patients identifiés ont été : la représentation du patient (s'en faire le porte-parole), le soutien éducationnel, la gestion personnalisée des symptômes, et l'intervention pivot.

Conclusion : Les IPO occupent un poste clé leur permettant d'adapter leur pratique aux besoins émotionnels et aux soins de soutien requis pour promouvoir la responsabilisation des patients et de leur famille.

Mots-clés : infirmières pivots, responsabilisation des patients, cancer, phase diagnostique

\section{INTRODUCTION}

T a phase diagnostique est une étape pénible pour les ـ patients. Vu la complexité du système de soins de santé, les patients doivent dialoguer avec beaucoup de professionnels

\section{AU SUJET DES AUTEURES}

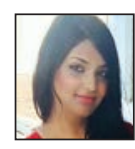

Gaya Jeyathevan, Ph.D.(c), Institut des politiques, de la gestion et de l'évaluation de la santé, Université de Toronto, Canada M5T $3 M 6$

Correspondance : Institut des politiques, de la gestion et de l'évaluation de la santé, Université de Toronto, Canada

Courriel : gaya.jeyathevan@mail.utoronto.ca

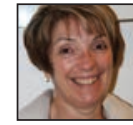

Manon Lemonde, inf. aut., Ph.D., Institut universitaire de technologie de l'Ontario, Oshawa, Canada L1H 7K4

Courriel : manon.lemonde@uoit.ca

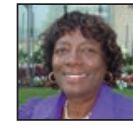

Angela Cooper Brathwaite, inf. aut., Ph.D., professeure adjointe, Institut universitaire de technologie de l'Ontario, Oshawa, Canada L1H 7K4

Courriel : angela.cooperbrathwaite@utoronto.ca

https://doi.org/10.5737/23688076272171177 de la santé répartis dans de multiples services de santé à l'intérieur du continuum des soins oncologiques. Moseholm et ses collègues (2016) signalent chez les patients un taux élevé d'angoisse et de dépression affectant la qualité de vie sur le plan de la santé durant l'évaluation diagnostique de cancer. Leurs travaux ont rapporté un pourcentage d'anxiété clinique avant l'obtention du diagnostic autour de $32 \%$ chez les patients cancéreux et de $35 \%$ chez les patients non cancéreux. D'autres chercheurs ont aussi constaté que les patients en attente d'un diagnostic de cancer connaissaient un risque accru de morbidité psychosociale (Bultz et Carlson, 2005; Drageset, 2012).

Durant le diagnostic de cancer, le traitement et les soins de suivi, la plupart des patients gagneront à être responsabilisés, car ils vivent continuellement des changements durant le long cheminement allant du diagnostic jusqu'aux soins palliatifs (Rosenbaum, Ernest et Spiegel, 2002). On décrit la responsabilisation du patient comme un processus au cours duquel les prestataires de soins de santé offrent au patient des choix et de l'information pour qu'il développe adresse et confiance. De simple receveur de soins passifs, il devient alors partenaire actif dans ses soins de santé (Organisation mondiale de la santé, 2009; European Network on Patient Empowerment, sans date). Il est essentiel que l'équipe de soins de santé conseille et encourage de manière continue les patients pour les responsabiliser. Toutefois, beaucoup de professionnels de la santé n'ont ni le temps ni l'expertise pour former et conseiller les patients (McCorkle et al., 2011).

L'intervention pivot en cancérologie est devenue un modèle de soins en Amérique du Nord pour promouvoir la responsabilisation des patients et de leur famille (Fillion et al., 2012). $\mathrm{Au}$ Canada, l'intervention pivot professionnelle en oncologie est habituellement exercée par des infirmières autorisées (Pedersen et Hack, 2010). Les infirmières pivots aident les patients et leur famille tout au long de la maladie en leur offrant un point de contact unique les reliant à l'équipe interdisciplinaire de soins de santé, au centre de cancérologie et aux services communautaires (Cook et al., 2013). Même si l'on reconnaît que l'infirmière pivot est un élément fondamental dans un réseau de soins oncologiques, l'intervention pivot n'est pas encore offerte de manière standardisée à l'échelle nationale (Action Cancer Ontario, 2010), et les recherches sur l'effet positif sur le vécu des patients des rôles joués par l'infirmière pivot sont rares (Cantril et Haylock, 2013; Fillion et al., 2012; Pedersen et Hack, 2011). À notre connaissance, aucune étude canadienne n'a porté sur la manière dont les infirmières pivots en oncologie (IPO) arrivent à responsabiliser les patients et leur famille durant la phase diagnostique de cancer. 
Le but de cette étude a donc été d'explorer les rôles joués par les IPO pour accroitre la responsabilisation des adultes atteints d'un cancer du poumon durant la phase diagnostique de soins oncologiques.

\section{CADRE CONCEPTUEL}

Les concepts du cadre bidimensionnel développé par Fillion et collègues (2012) sont à la base de cette étude; ils ont guidé l'analyse des données et l'interprétation des résultats. Le cadre bidimensionnel a été développé au début de 2012 afin de mieux comprendre les rôles joués par les infirmières pivots au Canada, de rendre ces dernières plus efficaces, et de les aider à mieux établir les priorités et à prendre des décisions tout en répondant aux attentes du système de soins de santé et des patients. Le cadre comporte deux dimensions théoriques : a) faciliter l'offre de soins continus; et b) promouvoir la responsabilisation des patients. La présente étude porte sur la deuxième dimension : promouvoir la responsabilisation des patients. Cette dimension englobe elle-même trois concepts : l'adaptation active, l'autogestion et les soins de soutien.

\section{MÉTHODES}

\section{Devis de l'étude}

Dans cette étude, nous avons fait appel à un protocole descriptif qualitatif pour explorer les rôles joués par les IPO afin d'accroître la responsabilisation d'adultes atteints d'un cancer du poumon traités au Centre de cancérologie régional de Durham, en Ontario, durant la phase

\begin{tabular}{|l|}
\hline $\begin{array}{l}\text { Tableau 1: Exemples de questions ouvertes tirées du Guide } \\
\text { d'entrevue }\end{array}$ \\
\hline Adaptation active \\
\hline $\begin{array}{l}\text { 1a. Version pour patients: Avez-vous reçu un enseignement ou un } \\
\text { soutien de la part de votre infirmière pivot pour savoir comment } \\
\text { mieux prendre soin de vous? Racontez un moment qui vous a aidé } \\
\text { à améliorer le sentiment que vous preniez bien soin de votre santé. } \\
\text { 1b. Version pour IPO : Quelles démarches actives entreprenez-vous } \\
\text { pour contribuer à la résolution du problème et à la prise de décision? }\end{array}$ \\
\hline
\end{tabular}

\section{Autogestion}

2a. Version pour patients : Quelle aide l'infirmière pivot vous a-telle apportée pour que vous puissiez vous adapter et gérer votre nouvel état de santé? Vos proches aidants (famille, amis) avaientils l'information requise sur votre santé et sur la façon de gérer vos symptômes pour arriver à vous aider?

2b. Version pour IPO : À quelle fréquence évaluez-vous ou surveillez-vous les symptômes des patients? De quelles façons offrez-vous une gestion des symptômes pour améliorer, dans l'ensemble, les soins oncologiques du patient et son expérience?

\section{Soins de soutien}

3a. Version pour patients: Votre infirmière pivot vous a-t-elle suggéré des services offerts dans votre communauté pour combler vos besoins en soins de soutien non satisfaits?

3b. Version pour IPO : Quels types de services offrez-vous aux patients pour combler leurs besoins physiques, émotionnels, psychosociaux, sociaux et spirituels? diagnostique. Ce protocole a été retenu parce qu'il permettait de présenter l'étude et ses résultats dans un langage vulgarisé (Sandelowski, 2000). Nous manquions par ailleurs d'études qualitatives sur les rôles joués par les infirmières pivots et sur leur impact durant la phase diagnostique de cancer. Le protocole de l'étude a combiné le recrutement d'un échantillon, une collecte de données, ainsi que des techniques d'analyse et de représentation. Des entrevues individuelles et un groupe de discussion avec questions ouvertes semi-structurées ont été organisés avec des patients et des IPO, respectivement. L'étude a été approuvée par les comités d'éthique sur la recherche de l'hôpital Lakeridge Health et de l'Institut universitaire de technologie de l'Ontario. Les participants ont signé un consentement éclairé avant les entrevues et le groupe de discussion.

\section{Recrutement}

Un échantillonnage de convenance de huit participants a été utilisé. Les IPO se sont appuyées sur les dossiers de patients et un tri téléphonique préliminaire pour repérer les patients potentiels qui répondaient aux critères d'admissibilité, soit : hommes et femmes adultes (18 ans et plus); aiguillés vers le programme dévaluation diagnostique du Centre de cancérologie régional de Durham en raison d'une présomption de cancer du poumon; ayant eu au moins deux échanges avec l'IPO avant la collecte de données; parlant un bon anglais. Une lettre d'invitation a été envoyée aux participants potentiels, puis les personnes intéressées qui répondaient aux critères de sélection ont été appelées par la chercheuse principale, qui leur a annoncé qu’elles étaient retenues pour l'étude. Les critères d'admissibilité suivants ont été établis pour les IPO : travailler au sein du programme d'évaluation diagnostique du Centre de cancérologie régional de Durham; avoir dispensé des soins de santé à des patients; avoir suivi avec succès des cours portant spécifiquement sur les poumons; avoir reçu la certification canadienne en soins infirmiers en oncologie $(\operatorname{CSIO}(\mathrm{C}))$. Le recrutement a été réalisé entre janvier 2013 et mars 2013. Il a pris fin lorsque les entrevues successives avaient atteint saturation, et qu'aucune nouvelle réponse ni nouveau thème n'émergeaient (Creswell, Plano Clark, Gutmann et Hanson, 2003).

\section{Collecte des données}

Chaque patient a été rencontré pendant 15 à 20 minutes pour répondre à des questions ouvertes. Les IPO participantes ont été réunies dans un groupe de discussion qui a duré environ 35 minutes pendant lequel elles ont répondu à des questions ouvertes. Les entrevues avec les patients et le groupe de discussion ont été animés par la chercheuse principale; cette dernière n'avait aucun lien préalable avec les participants. Le guide d'entrevue a été préparé en s'appuyant sur une description détaillée des concepts du cadre bidimensionnel. Les questions d'entrevue portaient sur des concepts comme : 1) l'adaptation active (p. ex. comment les IPO aidaient les patients à acquérir des mécanismes d'adaptation); 2) l'autogestion (p. ex. comment les IPO ont apporté du soutien aux patients pour qu'ils gèrent eux-mêmes leur santé altérée); et 3) les soins de soutien (p. ex. comment les IPO ont composé avec l'évolution des besoins des patients). Des questions exploratoires et récurrentes ont été utilisées en entrevues et en 
groupe de discussion pour explorer plus à fond des sujets particuliers et pour vérifier la compréhension qu'avait la personne interrogée de l'information recueillie (Patton, 2002). Les entrevues individuelles et les discussions de groupe ont été enregistrés et textuellement retranscrits. Le tableau 1 présente un échantillon des questions qui ont été posées.

\section{Analyse des données}

La collecte et l'analyse des données ont été effectuées de manière itérative. La chercheuse principale a vérifié l'exactitude et la précision des transcriptions. Des thèmes ont été établis à partir des récits des participants dans le cadre d'un processus itératif d'analyse thématique (Graneheim et Lundman, 2004). Un sous-ensemble de transcriptions d'entrevue a d'abord été codé par la chercheuse principale, qui a examiné attentivement toutes les données. Cette dernière a ensuite déterminé le codage pour le reste des transcriptions. Les deux autres chercheuses ont passé en revue les transcriptions, résumé la teneur informative des données pour identifier des thèmes, comparé l'exactitude des constats et l'interprétation des données, et exploré et résolu toutes incohérences dans le codage. Cette façon de faire assurait la rigueur et une plus grande réflexivité. Les segments de données, les idées et les mots véhiculant une idée ont été identifiés avant de créer les sous-ensembles thématiques. Les segments de données ont été regroupés par thèmes, puis les codes ont été regroupés en catégories et enfin, les grands thèmes ont été dégagés. Léquipe de recherche a testé auprès de sources extérieures ses analyses afin d'en maximiser la crédibilité et la fiabilité (Toma, 2011). Les trois chercheuses ont exploré divers plans thématiques jusqu'à atteindre le consensus et une pleine entente sur les thèmes retenus.

\section{CONSTATS}

Huit personnes ont participé à cette étude (quatre adultes atteints d'un cancer du poumon et ayant subi une ablation des lobes du poumon droit, et quatre infirmières pivots en oncologie). Le tableau 2 présente les caractéristiques des participants.

\section{Fonctions cliniques des infirmières pivots en oncologie}

La synthèse des résultats a permis d'identifier des fonctions cliniques fondamentales dans les rôles joués par les IPO pour accroître la responsabilisation des patients. Le tableau 3 présente les thèmes retenus accompagnés de citations des participants. Les fonctions cliniques fondamentales dans les rôles des IPO ont été déterminées sur la base des perceptions des patients quant à l'impact qu'ont eu les IP0 dans leur responsabilisation ainsi que celle de leur famille, particulièrement par rapport aux concepts d'adaptation active, d'autogestion et de soins de soutien. On a ainsi pu cerner que les fonctions suivantes augmentaient la responsabilisation du patient : se faire le porte-parole du patient en le représentant, fournir un soutien éducationnel, assurer une gestion personnalisée des symptômes, et agir comme intervenant pivot. Les deux premiers (représentation et soutien éducationnel) se rattachent à l'adaptation active, la gestion personnalisée des symptômes relève de l'autogestion, et le rôle d'intervenant pivot a été affilié aux soins de soutien.

\begin{tabular}{|c|c|}
\hline Caractéristiques des patients & $n=4 ; n, \dot{E}_{\text {cart }}$ \\
\hline \multicolumn{2}{|l|}{ Sexe } \\
\hline Homme & 2 \\
\hline Femme & 2 \\
\hline Âge & $58-71$ \\
\hline \multicolumn{2}{|l|}{ Symptômes vécus } \\
\hline Essoufflement & 2 \\
\hline Perte de poids & 2 \\
\hline Fatigue & 2 \\
\hline Enflure des jointures & 2 \\
\hline Aucun symptôme & 2 \\
\hline \multicolumn{2}{|l|}{ Éducation } \\
\hline Études secondaires & 3 \\
\hline Études universitaires (1er cycle)/cégep & 1 \\
\hline \multicolumn{2}{|l|}{ État civil } \\
\hline Marié & 3 \\
\hline Divorcé & 1 \\
\hline \multicolumn{2}{|l|}{ Statut professionnel } \\
\hline Retraité & 3 \\
\hline Travailleur & 1 \\
\hline Caractéristiques des infirmières pivots en oncologie & $n=4 ; n$, Écart \\
\hline \multicolumn{2}{|l|}{ Sexe } \\
\hline Homme & 0 \\
\hline Femme & 4 \\
\hline Âge & $39-50$ \\
\hline Années de service en oncologie & $4,5-20$ \\
\hline \multicolumn{2}{|l|}{ Éducation } \\
\hline Études universitaires/cégep & 3 \\
\hline Études universitaires ( $\left(2^{\mathrm{e}} / 3^{\mathrm{e}}\right.$ cycles $)$ & 1 \\
\hline \multicolumn{2}{|l|}{ Statut professionnel } \\
\hline Temps partiel & 2 \\
\hline Temps plein & 2 \\
\hline
\end{tabular}

\section{L'adaptation active}

L'un des rôles intrinsèques de l'IPO est de tenter dans une démarche proactive d'éliminer les facteurs de stress chez les patients durant la phase diagnostique. Elles y arrivent en assurant la représentation des patients et en offrant un soutien éducationnel, ce qui aide les patients à composer avec leur maladie.

La représentation des patients (s'en faire le porte-parole).

Il y a eu consensus entre les patients et les IPO participantes comme quoi les IPO joueraient un rôle important pour identifier les besoins des patients et de leur famille et ensuite s'en faire les porte-parole durant le processus diagnostique. 
Tableau 3: Thèmes et commentaires représentatifs des participants

\begin{tabular}{|c|c|}
\hline Thèmes & Source des commentaires représentatifs \\
\hline $\begin{array}{l}\text { Soutien } \\
\text { éducationnel }\end{array}$ & $\begin{array}{l}\text { Une IPO : } \\
\text { "L'infirmière consacre toujours du temps au patient pour passer en revue avec lui l'information fournie par le médecin et pour } \\
\text { examiner le résumé des examens qu'il pourrait avoir à subir." } \\
\text { Un patient: } \\
\text { "C'était tout un lot d'information. Elle en a passé en revue avec moi, surtout les tests, mais il y a toutes sortes de brochures sur le } \\
\text { cancer du poumon." }\end{array}$ \\
\hline $\begin{array}{l}\text { Gestion } \\
\text { personnalisée } \\
\text { des symptômes }\end{array}$ & $\begin{array}{l}\text { Des patients: } \\
\text { "Lorsque j'ai vu que j'avais des petites bosses dans la bouche, elle m'a expliqué que je pouvais utiliser du bicarbonate de soude ou } \\
\text { du rince-bouche, puis elle a ajouté qu'elle m'obtiendrait quelque chose pour cela. Elle m'a alors prescrit un rince-bouche. Elle était } \\
\text { là pour toutes ces petites choses. } \\
\text { "Elle nous a expliqué (au patient et à son épouse en rôle de proche aidant) comment prendre soin de moi, par exemple comment } \\
\text { éviter d'attraper le rhume; elle m'a aussi fait remarquer tout plein de petites choses. " }\end{array}$ \\
\hline $\begin{array}{l}\text { Intervention } \\
\text { pivot }\end{array}$ & $\begin{array}{l}\text { Une IPO : } \\
\text { "Bien des gens diagnostiqués d'un cancer, ou en attente de diagnostic de cancer, ne s'intéressent pas en premier lieu à cela. Ils } \\
\text { se demandent plutôt ce qui arrivera à leurs enfants, à leur travail... Ils ne songent pas à leur traitement. Il faut savoir s'intéresser } \\
\text { à leurs soucis et à leurs besoins du moment, qui parfois n'ont rien à voir avec le cancer. Le simple fait de savoir qu'une aide, des } \\
\text { ressources et des gens sont là facilite ce processus pour eux. } \\
\text { Un patient: } \\
\text { "Il y avait une telle variété de services offerts... Ç'a certainement comblé tous mes besoins. Je n'ai manqué de rien. J'ai appelé } \\
\text { pour qu'on m'emmène ici aujourd'hui." }\end{array}$ \\
\hline
\end{tabular}

Les IPO ont indiqué devenir « porte-parole du patient, afin qu'il puisse se rendre là où il a besoin d'être » dans les autres services hospitaliers ou services de soins de santé communautaires. Une participante a ajouté qu'en « représentant mes patients, je ne change pas le cours de leur maladie, mais je peux les aider à composer avec ce qu'ils vivent à ce moment ».

\section{Le soutien éducationnel.}

Les participants des deux groupes ont souligné le rôle important qu'ont pu jouer les IPO en offrant un soutien éducationnel permettant aux patients et à leur famille d'obtenir l'information et le soutien requis au moment de prendre des décisions vitales sur leurs soins de santé. A également été reconnu à titre de soutien éducationnel les explications détaillées sur les termes médicaux et les effets secondaires des traitements, qui viennent accroître les connaissances des patients quant aux tests effectués pendant la phase diagnostique. Grâce à ce soutien éducationnel, les patients ont dit s'être sentis plus à l'aise pour s'adapter activement à leur maladie et composer avec les changements vécus durant ce processus. Les IPO ont dit avoir offert un soutien éducationnel dès la première consultation afin que les patients soient en mesure de résoudre tout problème pouvant surgir et de prendre des décisions sur leurs soins de santé. Elles ont aussi indiqué qu'elles « demeuraient une ressource constante » pour les patients afin d'atténuer l'impact des facteurs de stress vécus par les patients et de les aider à prendre en main leur vie.

\section{L'autogestion}

L'autogestion s'est avérée un concept clé dans cette étude. Pour renforcer l'aptitude des patients à accepter leur maladie et à reprendre le contrôle de leur situation, les IPO ont pris l'initiative d'offrir une gestion personnalisée de leurs symptômes, ce qui les aide à composer avec les changements physiques. Les patients comprenaient leur état de santé et ses effets sur leurs corps. Ils avaient pu participer à la prise de décision avec les prestataires de soins de santé en contribuant au plan de soins et en faisant connaître leurs préférences. Ils se sentaient aussi mieux informés sur les choix et traitements offerts. 
La gestion personnalisée des symptômes.

Tous les patients participants ont dit que les IPO ont fait ce qu'il fallait pour s'assurer qu'ils maîtrisent des techniques pour composer plus efficacement avec le cancer (comme la gestion de leurs symptômes). Les patients ont également signalé que les IPO ont pris l'initiative d'expliquer aux membres de leur famille comment surveiller et gérer les symptômes, et comment les soigner comme il faut. Les deux groupes de participants ont rapporté qu'en plus de montrer des techniques de gestion de symptômes, les IPO avaient aussi évalué les symptômes à chaque consultation. Elles ont gardé un suivi des symptômes des patients en leur demandant de remplir une échelle de gestion des symptômes (la Edmonton Symptom Assessment Scale, ou EESE), et ont donné conseils et soutien aux patients sur la façon de gérer de manière proactive leur état altéré et les symptômes en fonction des résultats fournis par ces échelles. L'identification des besoins des patients s'est aussi révélée importante pour leur enseigner les diverses techniques leur permettant précisément de composer plus efficacement avec leur cancer et de planifier leurs soins en conséquence.

\section{Les soins de soutien}

Pour répondre aux besoins des patients et de leur famille en soins de soutien durant la phase diagnostique de soins oncologiques, les IPO ont offert une intervention pivot appropriée.

\section{L'intervention pivot.}

Les IPO ont souligné avoir cherché activement à cerner les besoins des patients en soins de soutien afin de pouvoir détecter la détresse générale et les besoins non comblés, ainsi que pour renforcer la responsabilisation. Par exemple, les IPO ont aiguillé des patients vers des ressources et services communautaires permettant de combler leurs besoins et ceux de leur famille. Tous les participants étaient d' avis que lorsqu'eux-mêmes ou leur famille avaient besoin de services précis, les IPO en facilitaient l'accès, que ce soit à l'hôpital ou dans les programmes communautaires. Les IPO ont perçu la chose comme un simple coup de main pour s'y retrouver dans les ressources, alors que les patients considéraient que leur IPO leur avait fourni des soins de soutien en les aiguillant vers des services communautaires.

\section{DISCUSSION}

Dans la présente étude, nous avons exploré les rôles joués par les infirmières pivots en oncologie pour accroître la responsabilisation d'adultes atteints d'un cancer du poumon durant la phase diagnostique de cancer. Quatre fonctions cliniques associées aux rôles joués par l'IPO pour accroître la responsabilisation des patients ont été identifiées à l'aide d'un protocole descriptif qualitatif : la représentation des patients en s'en faisant le porte-parole, le soutien éducationnel, la gestion personnalisée des symptômes, et l'intervention pivot. Ensemble, ces fonctions créent un modèle interconnecté qui place les patients atteints du cancer et les membres de leur famille au cœur des soins prodigués durant la phase diagnostique du continuum de la maladie. Cette étude qualitative se démarque du fait qu'elle s'appuie à la fois sur la perspective des patients atteints d'un cancer du poumon et sur celle des IPO afin de mieux comprendre les rôles que ces dernières peuvent jouer pour accroître la responsabilisation des patients durant la phase diagnostique de cancer.

Dans l'ensemble, cette étude a pu établir que les IPO servaient de pont entre le système de soins de santé et les patients. Selon Wilcox et Bruce (2010), le but principal du nouveau rôle d'IPO est de représenter les patients et leur famille en coordonnant et en rehaussant les soins entre tous les membres d'une équipe interdisciplinaire. Comme l'IPO possède une formation d'infirmière doublée d'une compréhension poussée des éléments d'oncologie et de la phase d'évaluation diagnostique, elle est des plus compétentes pour se faire la porte-parole des patients dont elle s'occupe. Les IPO ont expliqué qu'en maintenant de bonnes relations avec les patients et leur famille, elles étaient en mesure d'identifier les besoins et les préoccupations des patients et d'ainsi mieux représenter ces derniers. Elles ont indiqué que cette fonction de porte-parole se matérialisait surtout dans le rôle d'intermédiaire joué pour faciliter la communication entre le patient et le médecin. À plusieurs reprises, cette représentation a permis à l'IPO de diriger ses patients vers le service hospitalier dont ils avaient besoin. Deux patients ont aussi rapporté que les IPO les avaient aiguillés vers des services communautaires.

Les patients atteints du cancer ont en outre reçu beaucoup de matériel éducationnel durant leurs consultations, matériel décrit par certains comme étant « tout un lot d'information ». Weinman (1990) a fait ressortir que $75 \%$ des patients voulaient de l'information écrite sur leur maladie plutôt que de l'information verbale, car ils risquaient plus de mal comprendre ou d'oublier cette dernière. Les résultats de la présente étude apportent un nouvel éclairage. En effet, les patients interrogés ont fait l'éloge de l'IPO pour leur avoir expliqué clairement les renseignements présentés dans les livrets et autres brochures d'information. Ces derniers étaient utiles pour énoncer l'information, mais les patients voyaient leur anxiété et leur degré d'incertitude baisser lorsque l'IPO leur expliquait leur contenu, ce qui les aidait à mieux comprendre l'information. Le fait de donner de l'information en personne aux patients est venu enrichir les interactions personnelles entre l'IPO et les patients. Les soins oncologiques ont changé considérablement au cours des dernières décennies. Il existe actuellement une diversité de tests diagnostiques, de programmes d'évaluation, de nouveaux termes médicaux et de services de soins oncologiques accessibles. Les patients sont bombardés par nombre de renseignements et d'options. L'information écrite aide certes à diffuser l'information aux patients, mais elle ne s'avère peut-être pas aussi efficace qu'une discussion en personne avec l'IPO. De plus, dans la présente étude, les patients ont précisé qu'avoir une IPO comme soutien éducationnel leur avait permis de mieux comprendre certains renseignements médicaux connexes. Comme la littératie en matière de santé était un obstacle majeur pour les patients, les IPO se sont montrées des plus efficaces pour faire tomber cette barrière en offrant un complément d'information verbal durant les discussions en personne avec leurs patients. 
Les résultats de la présente étude ont montré que les IPO avaient non seulement éduqué sur la gestion des symptômes, mais avaient aussi veillé à évaluer et à surveiller les symptômes de leurs patients. L'IPO a d'abord fourni en temps opportun à ses patients un enseignement personnalisé sur la gestion des symptômes. Les patients interrogés ont expliqué que leur IPO leur avait fourni diverses ressources sur la gestion des symptômes et l'autogestion durant la première consultation. Ces ressources expliquaient comment composer avec les signes précurseurs résiduels et les signes de rechute, gérer les effets secondaires liés aux tests diagnostiques, respecter la médication et adopter au quotidien des routines d'autogestion. De plus, éduquer les patients quant à l'importance d'être attentif à des symptômes précis et de les gérer aura permis de réduire l'anxiété tout comme le temps d'attente pour des consultations médicales. En effet, les patients n'ont pas cherché à consulter leur médecin chaque fois qu'un symptôme se manifestait, car ils savaient comment les gérer de manière autonome à la maison. Le dernier énoncé indique que les patients ont pris en main leur santé et se sont servis de l'information fournie par l'IPO. Bien que les IPO aident les patients à se responsabiliser, cela s'est fait de manière limitée. Selon l'Organisation mondiale de la santé (2009), la responsabilisation se définit comme un processus dans le cadre duquel les patients comprennent leur rôle et reçoivent de leur prestataire de soins de santé des connaissances et des techniques qui leur permettent d'accomplir une tâche dans un environnement qui reconnaît la communauté et la diversité culturelle, et qui les invite à participer.

En plus d'éduquer à l'autogestion, les IPO ont évalué et surveillé les symptômes de leurs patients. En procédant à une évaluation des symptômes, elles ont mieux pu identifier chez les patients les symptômes importants qui nécessitaient une attention immédiate ou des changements dans le plan de traitement. Selon Yarbro, Frogge et Goodman (2004), des symptômes mal gérés peuvent affecter le traitement oncologique, le fonctionnement quotidien et la qualité de vie des personnes atteintes de cancer. À titre d'exemple, en surveillant une toux chronique persistante chez le patient, l'IPO peut informer le médecin si la toux s'aggrave, ce qui pourrait se traduire par d'autres séances de tomodensitométrie pour détecter tous changements aigus ou chroniques dans le parenchyme pulmonaire (Medic 8, sans date). Il était par conséquent essentiel pour les IPO d'aider leurs patients à composer avec la gestion des symptômes pouvant avoir un effet défavorable à la fois sur la qualité et la longévité de leur vie.

Les IPO ont aussi souligné que le fait de procéder à des dépistages, à des examens et à une évaluation de la condition des patients et de leurs progrès a été bénéfique, et a permis aux patients et à leur famille d'accéder à des services de soins de soutien. Par exemple, les IPO ont indiqué avoir fourni aux patients l'accès à des services communautaires comme des agences de soins à domicile ou des organismes communautaires (églises, organismes culturels connexes, etc.) pour combler leurs besoins physiques, psychosociaux, émotionnels et culturels. En fournissant un accès à ces services communautaires, les IPO ont pu responsabiliser les patients et leur famille afin qu'ils prennent en main la maladie et qu'ils s'impliquent dans leur communauté. Elles ont expliqué qu'offrir des soins de soutien a facilité la phase diagnostique chez les patients, car cela leur a fourni plus d'occasions de planifier leurs soins, d'organiser leur temps et de prendre leur vie et la maladie en main. Elles ont aussi expliqué que les patients ont ainsi pu se sentir soutenus par d'autres personnes dans leur communauté. Le fait de savoir que d'autres patients vivaient des situations semblables et recevaient des traitements similaires pour des cancers du même genre permettait d'avoir des gens auprès de qui chercher soutien et réconfort. Compte tenu de ces constats, on peut estimer que les besoins informationnels et émotionnels des patients ont été comblés par les IPO. À prime abord, les patients n'auraient pas forcément vu les ressources communautaires comme une priorité en raison du degré d'anxiété vécu durant la phase d'évaluation diagnostique (Drageset, 2012; Moseholm et al., 2016).

\section{Limites}

La présente étude accuse certaines limites. Bien que la taille de l'échantillon était petite, elle a permis à la chercheuse principale de procéder à des entrevues en profondeur, notamment avec les IPO, ce qui a mené à de nouveaux résultats sur le rôle des IPO. Nous avons aussi eu recours à deux différentes méthodes de collecte des données : des entrevues individuelles pour les patients et une discussion de groupe pour les IPO. La triangulation des données a fourni un meilleur éclairage du sujet et a renforcé la validité des données (Burns et Grove, 2009). Avec un échantillon plus grand, le recours à de multiples méthodes de collecte de données (entrevues individuelles et groupes de discussion) auprès des patients et des IPO aurait permis d'obtenir une analyse plus détaillée. Quoi qu'il en soit, les résultats de l'étude ont fourni de l'information sur la responsabilisation des patients et permis de tirer au clair les rôles que pouvaient jouer les infirmières pivots en oncologie.

Une autre limite était que seuls des participants d'expression anglaise ont participé à l'étude. L'exclusion de patients ne parlant pas couramment l'anglais a limité l'examen des perspectives linguistiques et culturelles dans les liens IPO-patients. Cependant, les IPO ont éliminé la barrière de la littératie sur la santé en offrant aux patients de l'information pertinente et en les incluant dans le processus de prise de décision pour les responsabiliser. Bien que dans cette étude on n'ait pas tenu compte du passé culturel des participants, il faudrait à l'avenir explorer dans le cadre d'études les barrières langagières et culturelles associées à d'autres contextes et cultures.

\section{Implications}

Il existe à l'heure actuelle un large éventail de directives et de normes de pratique destiné aux infirmières pour introduire les meilleures pratiques dans leur expertise. En ce qui a trait aux infirmières en oncologie évoluant dans un cadre canadien, les résultats de la présente étude ont soutenu et illustré la façon dont la responsabilisation des patients a été accrue dans un centre de cancérologie d'une collectivité. Les domaines de pratique de l'infirmière en oncologie, présentés dans le document « Normes de soins, rôles infirmiers en oncologie et compétences relatives aux rôles infirmiers » (2006) publié par l'Association canadienne des infirmières en oncologie (ACIO), 
valident les fonctions cliniques centrales du rôle de l'IPO identifiées dans la présente étude. Selon Jeyathevan, Lemonde, et Cooper-Brathwaite (2017), connaître les compétences centrales, les champs de pratique de l'infirmière pivot et le cadre d'intervention peut s'avérer utile pour les décideurs, gestionnaires et prestataires de soins de santé, afin de concevoir des rôles infirmiers semblables pour accompagner des patients souffrant de maladie mentale ou de maladies cardiovasculaires. Le cadre bidimensionnel décrit dans la présente étude pourrait aider à définir le contenu des modules de formation des infirmières, afin qu'elles offrent des soins continus et cohérents centrés sur les patients, et reçoivent une formation systématique reflétant les fonctions cliniques et les principaux domaines d'intervention de la responsabilisation des patients (Fillion et al., 2012). Enfin, il faudrait responsabiliser les patients pour qu'ils jouent un rôle plus actif dans leurs soins et dans leur prise de décision durant la phase diagnostique de cancer, même si cette période est pour eux éprouvante et angoissante.

\section{RÉFÉRENCES}

Action Cancer Ontario. (2010). Patient Navigation Pilot Project. Toronto, ON: Action Cancer Ontario.

Bultz, B.D. et Carlson, L.E. (1995). Emotional distress: The Sixth Vital Sign in Cancer Care. Journal of Clinical Oncology, 23, 6440-6441.

Canadian Association of Nurses in Oncology. (2006). Practice standards and competencies for the specialized oncology nurse. Toronto, ON: CANO/ACIO.

Cantril, C. et Haylock, P.J. (2013). Patient navigation in the oncology care setting. Dans Seminars in Oncology Nursing, 29(2), 76-90. WB Saunders.

Cook, S., Fillion, L., Fitch, M., Veillette, A.M., Matheson, T., Aubin, M. et Rainville, F. (2013). Principaux domaines d'exercice et compétences associées des infirmières œuvrant à titre de navigateurs professionnels en oncologie. Revue canadienne de soins infirmiers en oncologie, 23(1), 53-62. doi:10.5737/1181912x2314452

Creswell, J.W., Plano Clark, V.L., Gutmann, M.L. et Hanson, W.E. (2003). Advanced mixed methods research designs. Handbook of Mixed Methods in Social and Behavioral Research, 209-240.

Drageset, S. (2012). Psychological distress, coping and social support in the diagnostic and preoperative phase of breast cancer. Thèse de doctorat, Université de Bergen, Norway.

European Network on Patient Empowerment (sans date). Patient Empowerment_Living with Chronic Disease. Consulté sur http:// www.enope.eu/media/14615/a_series_of_short_discussion_ topics_on_different.pdf

Fillion, L., Cook, S., Veillette, A.M., De Serres, M., Aubin, M., Rainville, F., Fitch, M.I. et Doll, R. (2012a). La navigation professionnelle : une étude comparative de deux modèles canadiens. Revue canadienne de soins infirmiers en oncologie, 22(4), 267-277.

Fillion, L., Cook, S., Veillette, A.M., Aubin, M., De Serres, M., Rainville, F., Fitch, M.I. et Doll, R. (2012b). Professional navigation framework: Elaboration and validation in a Canadian context. Oncology Nursing Forum, 39(1), 58-69.

Graneheim, U.H. et Lundman, B. (2004). Qualitative content analysis in nursing research: Concepts, procedures and measures to achieve trustworthiness. Nurse Education Today, 24(2), 105-112.

Jeyathevan, G., Lemonde, M. et Cooper Brathwaite, A. (2017). Rôle des infirmières pivots en oncologie pour assurer la continuité des soins offerts aux adultes durant la phase diagnostique du cancer $\mathrm{du}$ poumon. Revue canadienne de soins infirmiers en oncologie, 27(1), 81-87.

\section{CONCLUSION}

Comme les besoins des personnes atteintes du cancer sont complexes, il faut des professionnels de la santé qui servent d'intervenants pivots pour aiguiller les patients et leur famille dans les rouages du système de santé, afin de surmonter les obstacles que présentent les facteurs liés aux paliers du système de santé. Le rôle de l'infirmière pivot en oncologie continue de se développer et déprendra de la collaboration régulière des professionnels de la santé et de leur communication. Cette étude a montré que les IPO peuvent adapter leur pratique aux besoins émotionnels et aux besoins de soutien des patients, de manière à les responsabiliser, eux et leur famille. Par conséquent, il faudrait entrevoir une collaboration entre les professionnels de la santé pour examiner le fonctionnement d'une équipe interdisciplinaire dans une perspective globale et pour voir comment cela affecte la norme de soins pour les patients et leur famille à l'intérieur d'un continuum de soins oncologiques.

Medic 8. (sans date). CT Scan. Consulté sur http://www.medic8.com/ healthguide/articles/ctscan.html

McCorkle, R., Ercolano, E., Lazenby, M., Schulman-Green, D., Schilling, L.S., Lorig, K. et Wagner, E.H. (2011). Self-management: Enabling and empowering patients living with cancer as a chronic illness. CA: A Cancer Journal for Clinicians, 61(1), 50-62.

Moseholm, E., Rydahl-Hansen, S., Overgaard, D., Wengel, H., Frederiksen, R., Brandt, M. et Lindhardt, B. (2016). Health-related quality of life, anxiety and depression in the diagnostic phase of suspected cancer and the influence of diagnosis. Health Quality Life Outcomes, 14(80). doi:10.1186/s12955-016-0484-9

Organisation mondiale de la santé. (2009). WHO guidelines on hand hygiene in health care: First global patient safety challenge. Clean care is safer care. 2, Patient empowerment and health care. Organisation mondiale de la santé.

Patton, M.Q. (2015). Qualitative research and evaluation methods: Integrating theory and practice. SAGE Publications Inc.

Pedersen, A. et Hack, T.F. (2010). Pilots of oncology health care: A concept analysis of the patient navigator role. Oncology Nursing Forum, 37(1), 55-60. doi:10.1188/10.ONF.55-60

Pedersen, A.E. et Hack, T.F. (2011). The British Columbia Patient Navigation Model: A critical analysis. Dans Oncology Nursing Forum, 38(2).

Rosenbaum, E.H. et Spiegel, D. (2002). Patient Empowerment through Supportive Care. Cancer Supportive Care Programmes.

Sandelowski, M. (2000). Focus on research methods. What happened to qualitative description? Research in Nursing and Health, 23, 334-240.

Toma, J.D. (2011). Approaching rigor in applied qualitative research. The SAGE Handbook for Research in Education: Pursuing Ideas as the Keystone of Exemplary Inquiry, 405-423.

Weinman, J. (1990). Providing written information for patients: Psychological considerations. Journal of the Royal Society of Medicine, 83(5), 303-305.

Wilcox, B. et Bruce, S.D. (2010). Patient navigation: A “win-win” for all involved [Abstract]. Oncology Nursing Forum, 37(1), 21-25.

Yarbro, C.H., Frogge, M.H. et Goodman, M. (2004). Cancer symptom management ( $3^{e}$ éd.). Sudbury, MA : Jones and Bartlett Publishers.

Zabora, J., BrintzenhofeSzoc, K., Curbow, B., Hooker, C. et Piantadosi, S. (2001). The prevalence of psychological distress by cancer site. Psycho-Oncology, 10(1), 19-28. 